\title{
TRIAGEM E ATIVAÇÃO DA VIA VERDE dO ACIDENTE VASCULAR CEREBRAL: DIFICULDADES SENTIDAS PELOS ENFERMEIROS
}

TRIAJE Y ACTIVACIÓN DEL PROTOCOLO CÓDIGO ICTUS: DIFICULTADES QUE SIENTEN LOS ENFERMEROS

TRIAGE AND ACTIVATION OF THE CODE STROKE PROTOCOL: DIFFICULTIES EXPERIENCED BY NURSES

DOI 10.33194/rper.2020.v3.n2.14.5829 | Submetido 13/11/2020 | Aprovado 15/12/2020

\author{
Anna Caroline Leite Costa $^{3}$ iD; Leonel São Romão Preto ${ }^{1}$ (D); Ilda Maria Morais Barreira ${ }^{2}$ (D); Léia Arcanjo Mendes ${ }^{4}$ iD; \\ Fernanda Lopes de Araújo ${ }^{3}$; ; André Filipe Morais Pinto Novo ${ }^{1}$ iD \\ 1 - Escola Superior de Saúde do Instituto Politécnico de Bragança; 2 - Unidade Local de Saúde do Nordeste; \\ 3 - Universidade Federal de Minas Gerais; 4 - Unimed Belo Horizonte
}

\section{RESUMO}

Enquadramento: 0 processo de triagem contribui para o tratamento precoce do Acidente Vascular Cerebral (AVC) reduzindo a mortalidade e a incapacidade.

Objetivo: Analisar as dificuldades dos enfermeiros na realização da triagem e ativação da via verde do AVC.

Método: Estudo descritivo e transversal. Os dados foram colhidos através de questionário, por amostragem de conveniência, num serviço de urgência médico-cirúrgica.

Resultados: A amostra incluiu 21 enfermeiros (39,7 $\pm 7,4$ anos), a maioria mulheres $(71,4 \%)$. 0 tempo médio de serviço em urgência era de 9,5 ( $\pm 6,2$ anos).

A violência verbal e física de utentes ou familiares foi a dificuldade mais referida relativamente à triagem. Cerca de $29 \%$ concordam parcial e $19 \%$ totalmente que a informação inadequada sobre a hora de início dos sintomas constitui uma dificuldade aquando da ativação da via verde do AVC.

Conclusão: A triagem e o protocolo da via verde são vistos como facilitadores na assistência ao AVC, com dificuldades pontuais em algumas fases do processo.

Palavras-chave: acidente vascular cerebral; triagem; serviço de urgência; avaliação em enfermagem

\section{RESUMEN}

Marco contextual: El proceso de triaje contribuye al tratamiento precoz del ictus, reduciendo la mortalidad y la discapacidad.

Objetivo: Analizar las dificultades en el triaje y activación del protocolo de Código Ictus.

Método: Estudio descriptivo y transversal. Los datos fueron recolectados a través de cuestionario, por muestreo de conveniencia, en un servicio de emergencia médico-quirúrgico.

Resultados: La muestra incluyó 21 profesionales de enfermería $(39,7 \pm 7,4$ años), la mayoría mujeres ( $71,4 \%)$. El tiempo promedio de trabajo en urgencias fue de 9,5 ( $\pm 6,2$ años).

La violencia verbal y física de pacientes y familiares fue la dificultad más mencionada, en cuanto al triaje. Alrededor del $29 \%$ coincide parcial y el $19 \%$ totalmente en que la información inadecuada sobre el momento de inicio de los síntomas constituye una dificultad para activar el Código Ictus.

Conclusión: El triaje y el protocolo Código Ictus se consideran facilitadores asistenciales, con dificultades esporádicas en algunas fases del proceso.

Palabras clave: accidente cerebrovascular; triaje; servicio de urgencia en hospital; evaluación en enfermería

\section{ABSTRACT}

Background: The triage process contributes to early stroke treatment, reducing mortality and disability.

Objective: To analyze nurses' difficulties in triage and activating the Code Stroke protocol.

Method: Descriptive and cross-sectional study. The data were collected, by convenience sampling, in an emergency medical-surgical service through a questionnaire.

Results: The sample included 21 nurses (39.7 \pm 7.4 years), mostly women $(\mathbf{7 1 . 4 \% )}$. In terms of professional experience, the working time in emergency department was 9.5 ( \pm 6.2 years).

The verbal and physical violence of patients and family members was the most mentioned difficulty regarding triage. About $29 \%$ partially and $19 \%$ fully agree that inadequate information about two symptoms at the time of onset constitutes a difficulty in activating the Code Stroke. 
Conclusion: Triage and the Stroke Code protocol are considered facilitators of care, with sporadic difficulties in some phases of the process.

Keywords: stroke; triage; emergency service hospital; nursing assessment

\section{INTRODUÇÃO}

Nas últimas décadas houve um aumento da expectativa de vida da população mundial e as principais causas de morbimortalidade, outrora caracterizadas pelas doenças infeciosas, foi gradualmente cedendo espaço para as doenças crónicas não transmissíveis, entre elas as doenças cérebro-cardiovasculares como o Acidente Vascular Cerebral (AVC) ${ }^{(1,2)}$. Esta doença é considerada a segunda maior causa de mortalidade em todo o mundo e a primeira causa de incapacidade neurológica ${ }^{(3)}$. Por outro lado as pessoas que sobrevivem ao AVC apresentam grande fragilidade, limitação para 0 desempenho de atividades de vida diária e uma menor esperança de vida ${ }^{(4,5)}$.

Em Portugal, a incidência do AVC é uma das mais altas entre os países europeus, e é a principal causa de mortalidade no país, ocasionando cerca de $11 \mathrm{mil}$ mortes por ano ${ }^{(6)}$. Considerando a elevada mortalidade $\mathrm{e}$ as possíveis sequelas resultantes do AVC, o reconhecimento precoce dos sintomas e a agilidade no atendimento nos serviços de urgência contribuem para melhores prognósticos ${ }^{(1)}$.

Tendo em conta a eficiência do processo de atendimento, em 1994 foi desenvolvido um protocolo de triagem de prioridades em urgência, denominado Sistema de Triagem de Manchester (STM), implementado em Portugal a partir de $2005^{\left({ }^{(7)}\right.}$. Por meio deste sistema é possível a identificação das prioridades no atendimento e a subsequente definição dos tempos limites recomendados até à avaliação clínica. O STM tem sido utilizado em muitos países, podendo ser realizado por médicos ou enfermeiros habilitados - mais frequentemente enfermeiros (8,9). Segundo alguns autores estes últimos profissionais, ao direcionar a sua avaliação para a pessoa com sinais e sintomas e não para um possível diagnóstico clínico, são os que melhor se adequam a esta ferramenta ${ }^{(10)}$. Na mesma linha, a visão holística do enfermeiro, permite o estabelecimento de uma relação empática, valorizando não só os aspetos biológicos, mas também os sociais e psicológicos ${ }^{(10)}$.

Entre as dificuldades encontradas pelos profissionais que realizam o processo de triagem, destacam-se a insatisfação decorrente da tarefa; a elevada afluência de doentes em relação à capacidade do serviço; alterações no estado de saúde do doente em consequência do tempo de espera; o questionamento médico da avaliação realizada pelo enfermeiro; a dificuldade na descrição de queixas por parte dos doentes e ainda a violência física e/ou verbal por parte de acompanhantes e doentes ${ }^{(11-14)}$. Um estudo realizado em Portugal sobre as dificuldades percecionadas pelos enfermeiros triadores e o seu grau de satisfação com o processo de triagem concluiu que os enfermeiros percecionam mais dificuldades em lidar com as queixas dos doentes relativamente ao tempo de espera para atendimento e com o facto de os médicos questionarem o seu desempenho na triagem ${ }^{(15)}$.

A avaliação inicial do doente com suspeita de AVC conta ainda com a possibilidade de ser ativado um protocolo específico de emergência, em Portugal designado por Via Verde do AVC (VV do AVC) e na literatura anglosaxónica por Code Stroke ${ }^{(16)}$. A VV do AVC tem como objetivo que todos os doentes com AVC isquémico tenham acesso a um diagnóstico rápido e, nos casos elegíveis, a tratamento de reperfusão precoce ${ }^{(17)}$. As terapias de reperfusão (fibrinólise e trombectomia) melhoram o fluxo sanguíneo cerebral contribuindo para a viabilidade do tecido isquémico nas zonas periféricas à lesão, melhoram a recuperação funcional do AVC isquémico e os seus benefícios clínicos encontram-se suportados por um nível de evidência $A^{(18)}$.

Pelo acima exposto concluímos que a avaliação inicial e triagem das pessoas com AVC que recorrem a um serviço de urgência é de extrema importância, podendo este processo influenciar os resultados em saúde e o desfecho final, relativamente à mortalidade, funcionalidade e qualidade de vida. Considerando que essa avaliação inicial é na maioria das vezes realizada pelo enfermeiro é importante compreender as suas dificuldades e limitações na execução desta atividade assistencial. No entanto, e até onde chega o conhecimento bibliográfico dos autores da presente investigação, são escassos os estudos que abordaram as perceções e as dificuldades sentidas pelos enfermeiros no atendimento de urgência às pessoas com AVC, designadamente no que se prende com os processos inerentes ao STM e VV do AVC. Assim a investigação que ora se apresenta teve como objetivo geral analisar as dificuldades sentidas pelos enfermeiros que trabalham num serviço de urgência durante o processo de triagem e ativação do protocolo da via verde do AVC.

\section{MÉTODO}

Tendo em conta o objetivo geral desenhou-se um estudo descritivo e transversal, com uma abordagem quantitativa $o$ qual foi realizado numa unidade hospitalar do norte de Portugal que atende uma população residente de mais de 135000 habitantes.

A amostra do estudo foi composta por todos os profissionais de enfermagem que desempenham funções no Serviço de Urgência Médico-Cirúrgica da instituição alvo do estudo e que, tendo formação específica, cumpriam o critério de inclusão de fazerem habitualmente triagem e deste modo poderem também ativar a VV do AVC $(n=21)$.

A colheita de dados ocorreu por meio da aplicação de um questionário estruturado e desenvolvido pelos pesquisadores, após prévio levantamento de dados bibliográficos acerca das principais dificuldades enfrentadas pelos profissionais no processo de triagem, especialmente do AVC $(11,12,15,16,19)$. Em seguida, este foi 
revisto por três enfermeiros especialistas (um em enfermagem médico-cirúrgica, outro em enfermagem comunitária e ainda outro em enfermagem de reabilitação), que, de forma independente, avaliaram o instrumento e fizeram adequações gramaticais e de conteúdo necessárias para a melhor compreensão dos respondentes.

O instrumento de colheita de dados foi dividido em duas partes; sendo que a primeira contemplou dados demográficos e o processo de formação e experiência profissional, enquanto que a segunda parte contemplou aspetos relacionados com o processo de triagem, totalizado 20 questões tipo Likert. Dessas questões, 16 abordavam as dificuldades encontradas pelos profissionais no processo de triagem por meio do STM, enquanto as demais abordavam as especificidades na triagem do paciente com suspeita de AVC. Para cada questão, foram apresentadas cinco alternativas de resposta: (a) Discordo totalmente (b) Discordo parcialmente (c) Não concordo nem discordo (d) Concordo parcialmente (e) Concordo totalmente. Tais alternativas permitem identificar os diferentes níveis de intensidade de opinião a respeito de uma determinada temática ${ }^{(20)}$.

Objetivando a minimização de erros, o instrumento de recolha de dados foi aplicado pelo mesmo investigador a todos os profissionais de enfermagem, obedecendo a uma marcação prévia, antes do início dos turnos de trabalho. Todos os participantes assinaram o Termo de Consentimento Livre e Esclarecido, e seu anonimato foi preservado. O projeto do estudo foi submetido à Comissão de Ética da ULSNE, tendo obtido parecer positivo com número de apreciação 2017/1094.

As informações recolhidas foram armazenadas eletronicamente num ficheiro de dados do programa SPSS (Statistical Package for Social Sciences) versão 19, onde se efetuou o seu processamento estatístico, sendo obtidas as frequências absolutas e relativas das variáveis.

\section{RESULTADOS}

Foram analisados 21 questionários aplicados aos enfermeiros com experiência nos protocolos STM e VVAVC. Entre os respondentes, 71,4\% eram do sexo feminino e os restantes $28,6 \%$ pertenciam ao sexo masculino. Em relação à idade, a média foi de 39,7 anos (com desvio padrão 7,3 anos). Relativamente à experiência profissional os enfermeiros tinham um tempo de serviço em média de 10,5 anos (para um desvio padrão de 6,0 anos).

Especificamente no serviço de urgência médicocirúrgica, e em termos médios, o tempo de serviço foi de 9,5 anos (desvio padrão 6,2 anos). Quanto ao tempo decorrido desde a última formação ou reciclagem nos fluxogramas do STM a média situou-se nos 7,4 anos (desvio padrão 4,3 anos).

Quanto aos resultados obtidos nos aspetos relacionados com o processo de triagem os mesmos encontram-se descritos na Tabela 1. Como se denota uma percentagem significativa de enfermeiros concordam parcialmente $(28,6 \%)$ ou concordam totalmente $(33,3 \%)$ com a afirmação de se sentirem confortáveis em executar a atividade de triagem. Em sentido oposto 8 elementos referiram sentirem algum desconforto com a atividade da triagem.

O processo de triagem não parece constituir uma dificuldade para a maioria dos participantes do estudo, conforme podemos observar pelos resultados obtidos relativamente à segunda afirmação. Por outro lado o receio em atrasar o acesso de um utente ao serviço obtém a concordância parcial de cerca de $24,0 \%$ dos enfermeiros. Salientamos ainda o facto de que obtém elevados níveis de concordância, parcial $(38,1 \%)$ ou total $(19,0 \%)$, o enunciado de que a violência verbal e física dos utentes e seus familiares é uma dificuldade para o enfermeiro de triagem.

Na Tabela 2 apresentam-se os dados relativamente ao grau de concordância com afirmações sobre o protocolo da VV do AVC. A maioria dos participantes $(71,4 \%)$ discordam totalmente que os critérios de ativação deste protocolo constituam uma dificuldade para eles. $\mathrm{Na}$ mesma linha a gravidade do quadro do doente $(57,1 \%)$ ou as possíveis complicações do quadro clínico $(61,9 \%)$ não parecem ser uma dificuldade para a maioria dos inquiridos no momento da triagem. Por outro lado cerca de 29,0\% concordam parcialmente e 19,0\% totalmente que a informação inadequada sobre a hora de início dos sintomas constitui uma dificuldade aquando do processo de ativação da VV do AVC.

\section{DISCUSSÃO}

Em relação ao perfil dos participantes a maioria pertencia ao sexo feminino, com uma média de idade de 39,7 anos. Perfil similar foi descrito numa pesquisa realizada na região norte de Portugal que objetivou avaliar a satisfação dos enfermeiros com o STM, na qual $68,1 \%$ dos profissionais eram do sexo feminino e cuja média de idades rondou os 41 anos ${ }^{(21)}$. Sabe-se que a enfermagem é uma profissão onde ainda predomina o género feminino e que essa taxa de feminização está sobretudo ligada a questões históricas e culturais ${ }^{(22)}$.

Deparamo-nos com uma amostra cujos elementos apresentam elevada experiência profissional em urgência traduzido pelo tempo médio de 9,5 anos em que trabalham neste serviço. 0 enfermeiro triador deve ser um profissional qualificado já que desempenha um papel fundamental na avaliação, priorização do atendimento, identificação e encaminhamento dos doentes. A experiência profissional pode contribuir para a melhoria da tomada de decisão no momento da triagem. Um estudo prévio concluiu que à medida que aumenta o tempo de experiência tende a diminuir a perceção das dificuldades em triagem (15). Sabe-se ainda que a atuação deste profissional no processo de triagem pode impactar no desfecho clinico. Por isso, recomenda-se que tal atividade seja executada por profissionais com experiência, de modo a realizar a triagem com segurança e agilidade ${ }^{(14)}$. 
Tabela 1 - Dificuldades encontradas pelos profissionais na triagem de utentes pelo Protocolo de Manchester

\begin{tabular}{|c|c|c|c|c|c|}
\hline \multirow{3}{*}{ Dificuldades encontradas pelos profissionais } & \multicolumn{5}{|c|}{ Taxa de concordância } \\
\hline & ${ }^{*} \mathrm{~A}$ & ${ }^{* *} B$ & ${ }^{* * *} \mathrm{C}$ & ${ }^{\#} \mathrm{D}$ & ${ }^{\# \#} \mathbf{E}$ \\
\hline & $n(\%)$ & $n(\%)$ & $n(\%)$ & $n(\%)$ & $n(\%)$ \\
\hline Sinto-me confortável ao executar a atividade de triagem & $3(14,3)$ & $5(23,8)$ & $0(0,0)$ & $6(28,6)$ & $7(33,3)$ \\
\hline $\begin{array}{l}\text { O processo de triagem pelo Sistema de Triagem Manchester é uma } \\
\text { dificuldade para mim }\end{array}$ & $10(47,6)$ & $9(42,9)$ & $0(0,0)$ & $2(9,5)$ & $0(0,0)$ \\
\hline 0 processo de triagem informatizado é uma dificuldade para mim & $13(61,9)$ & $5(23,8)$ & $2(9,5)$ & $1(4,8)$ & $0(0,0)$ \\
\hline Executar outras tarefas, além da triagem, é uma dificuldade para mim & $13(61,9)$ & $4(19,0)$ & $3(14,3)$ & $1(4,8)$ & $0(0,0)$ \\
\hline $\begin{array}{l}\text { A insegurança que sinto em realizar a triagem é uma dificuldade para } \\
\text { mim }\end{array}$ & $11(52,4)$ & $6(28,6)$ & $2(9,5)$ & $2(9,5)$ & $0(0,0)$ \\
\hline A minha inexperiência na triagem é uma dificuldade para mim & $14(66,7)$ & $(9,5)$ & $2(9,5)$ & $3(14,3)$ & $0(0,0)$ \\
\hline $\begin{array}{l}\text { O receio em atrasar o acesso de um utente ao serviço é uma dificuldade } \\
\text { para mim }\end{array}$ & $9(42,7)$ & $4(19,0)$ & $3(14,3)$ & $5(23,8)$ & $0(0,0)$ \\
\hline O perfil clínico dos utentes é uma dificuldade para mim & $8(38,1)$ & $4(19,0)$ & $6(28,6)$ & $3(14,3)$ & $0(0,0)$ \\
\hline $\begin{array}{l}\text { O número elevado de utentes em relação à real capacidade do serviço é } \\
\text { uma dificuldade para mim }\end{array}$ & $5(23,8)$ & $4(19,0)$ & $6(28,6)$ & $3(14,3)$ & $3(14,3)$ \\
\hline $\begin{array}{l}\text { O questionamento da classificação pelo profissional médico é uma } \\
\text { dificuldade para mim }\end{array}$ & $7(33,3)$ & $2(9,5)$ & $\begin{array}{c}10 \\
(47,6)\end{array}$ & $1(4,8)$ & $1(4,8)$ \\
\hline $\begin{array}{l}\text { O questionamento da classificação por parte dos utentes é uma } \\
\text { dificuldade para mim }\end{array}$ & $5(23,8)$ & $7(33,3)$ & $8(38,1)$ & $1(4,8)$ & $0(0,0)$ \\
\hline $\begin{array}{l}\text { O questionamento da classificação por outros profissionais de } \\
\text { enfermagem é uma dificuldade para mim }\end{array}$ & $4(19,0)$ & $4(19,0)$ & $\begin{array}{c}10 \\
(47,6)\end{array}$ & $3(14,3)$ & $0(0,0)$ \\
\hline $\begin{array}{l}\text { O questionamento dos utentes quanto ao tempo de demora no } \\
\text { atendimento e à falta de informação acerca da classificação atribuída é } \\
\text { uma dificuldade para mim }\end{array}$ & $5(23,8)$ & $6(28,6)$ & $6(28,6)$ & $4(19,0)$ & $0(0,0)$ \\
\hline $\begin{array}{l}\text { As queixas inespecíficas apresentadas pelos utentes são uma dificuldade } \\
\text { para mim }\end{array}$ & $3(14,0)$ & $9(42,7)$ & $2(9,5)$ & $6(28,6)$ & $1(4,8)$ \\
\hline $\begin{array}{l}\text { O agravamento do estado de saúde de um paciente em decorrência do } \\
\text { tempo de espera é uma dificuldade para mim }\end{array}$ & $4(19,0)$ & $\begin{array}{c}10 \\
(47,6)\end{array}$ & $0(0,0)$ & $7(33,0)$ & $0(0,0)$ \\
\hline $\begin{array}{l}\text { A violência verbal e física dos utentes e seus familiares é uma dificuldade } \\
\text { para mim }\end{array}$ & $1(4,8)$ & $5(23,9)$ & $3(14,3)$ & $8(38,1)$ & $4(19,0)$ \\
\hline
\end{tabular}

Tabela 2 - Dificuldades encontradas pelos profissionais na ativação do protocolo da via verde do Acidente Vascular

\section{Taxa de concordância}

\section{Dificuldades encontradas pelos profissionais}

Os critérios de ativação da via verde do AVC são uma dificuldade para mim
A gravidade do quadro dos utentes com AVC é uma dificuldade para mim
As possíveis complicações de um AVC são uma dificuldade para mim no momento da triagem
A informação inadequada relativamente ao tempo de manifestação dos sintomas é uma dificuldade para mim

\begin{tabular}{|c|c|c|c|c|}
\hline${ }^{*} \mathrm{~A}$ & ${ }^{* *} \mathrm{~B}$ & ${ }^{* * *} \mathrm{C}$ & ${ }^{\#} \mathrm{D}$ & ${ }^{\# \#} \mathrm{E}$ \\
\hline $\mathbf{n ( \% )}$ & $\boldsymbol{n}(\%)$ & $n(\%)$ & $n(\%)$ & $n(\%)$ \\
\hline $12(71,4)$ & $1(4,8)$ & $0(0,0)$ & $4(19,0)$ & $1(4,8)$ \\
\hline $13(61,9)$ & $3(14,3)$ & $2(9,5)$ & $2(9,5)$ & $1(4,8)$ \\
\hline $6(28,6)$ & $3(14,3)$ & $2(9,5)$ & $6(28,6)$ & $4(19,0)$ \\
\hline
\end{tabular}

No nosso estudo o tempo médio decorrido desde a formação ou reciclagem em triagem cifrou-se em 7,4 anos. Um estudo realizado no Centro Hospitalar
Universitário de Coimbra encontrou que 58,5\% dos enfermeiros tinha realizado o curso de triagem há mais de 10 anos e $39,6 \%$ entre 5 a 10 anos ${ }^{(23)}$. É importante 
referir que os profissionais responsáveis pelo acolhimento e triagem de doentes em serviços de urgência necessitam aprimorar com mais continuidade os seus conhecimentos e habilidades com vista à melhoria da avaliação e classificação de prioridades. De salientar, por outro lado, que o STM é sujeito a auditorias regulares cujo objetivo não se limita à identificação de falhas e problemas mas também promover a comunicação, acolhendo dúvidas e sugestões, assumindo igualmente deste modo um carater formativo ${ }^{(23)}$.

Quanto à execução da triagem, observou-se que a maioria dos profissionais $(61,9 \%)$ relata estar parcial ou totalmente satisfeito na realização da tarefa. No entanto $38,1 \%$ dos respondentes referem desconforto na execução da atividade. A atividade dos enfermeiros na classificação de risco é complexa, requerendo agilidade, raciocínio clínico, destreza e boa capacidade de comunicação com utentes e familiares. Por isso, em certos cenários é observado algum nível de insatisfação de uma parcela de enfermeiros triadores ${ }^{(10,21)}$.

Em relação à triagem por meio do STM, evidenciou-se que a maior parte dos profissionais não percebem a ferramenta, mesmo de modo informatizada, como um dificultador na classificação de risco. Este achado corrobora com outros estudos que evidenciam elevada aceitação do STM pelos enfermeiros, reconhecendo-o como um método confiável para a classificação de prioridades no atendimento $(10,21,24)$. Estudos prévios destacam um excelente índice de concordância interobservador quando a triagem é realizada por enfermeiros, revelando-se a ferramenta muito eficaz na priorização da assistência em saúde, identificando os doentes em situação de maior risco ${ }^{(24)}$.

A maioria dos participantes do nosso estudo não sente que a insegurança ou a inexperiência sejam fatores que dificultem o processo de triagem. Estes achados vão de encontro à literatura que aponta que o enfermeiro com experiência em triagem geralmente tem segurança em suas condutas e decisões ${ }^{(10,21,24)}$. Alguns autores referem que a segurança sentida pelos profissionais durante a triagem advém das características intrínsecas ao protocolo de Manchester, designadamente dos seus discriminadores e fluxogramas, que respaldam a classificação realizada pelo profissional ${ }^{(10,21,24)}$.

Quanto ao questionamento de outros profissionais de saúde acerca da classificação atribuída, ressalta-se que no presente estudo tal situação não foi apontada como fator dificultador do processo de triagem para a maioria dos enfermeiros. No entanto, sabe-se que a discordância entre a classificação atribuída é um problema recorrente nos serviços de urgência, resultado por vezes em conflitos. A este respeito, o trabalho em equipa e a boa comunicação interdisciplinar emergem como estratégias organizacionais facilitadoras da resolução de conflitos e da atividade assistencial (16), já que o sistema de triagem deve potenciar a uniformidade consistente de critérios ao longo do tempo ${ }^{(13)}$.

No presente estudo a violência física ou verbal por parte de utentes ou acompanhantes foi relatada como uma dificuldade significativa. Estudos anteriores apontam que a violência, especialmente verbal, é frequente em serviços de urgência, em especial direcionada ao profissional da triagem. Os conflitos e violência experienciada pelos enfermeiros podem resultar em stress, insegurança, medo, ansiedade e absenteísmo laboral $(25,26)$.

Relativamente aos critérios de ativação da via verde do AVC estes não constituem uma dificuldade para a maioria dos enfermeiros. A ativação da VV do AVC é feita independentemente da classificação de prioridades atribuída pelo Sistema de Triagem de Manchester. Uma explicação plausível para a baixa dificuldade em ativar a VV do AVC por parte dos nossos participantes poderá residir no facto de a mesma se encontrar consolidada no nosso país, decorrido mais de uma década, desde a sua implementação em $2007^{(27)}$. Estudos prévios realizados na zona norte de Portugal apontam para uma elevada taxa de ativação da VV, chegando a abranger $16,3 \%$ de todos os doentes com AVC ${ }^{(27)}$ e especificamente $35,9 \%$ de todos os AVC de origem isquémica ${ }^{(16)}$.

Nem a gravidade do quadro do AVC, nem as possíveis complicações constituem uma dificuldade para a maioria dos enfermeiros triadores. A ativação do protocolo intra-hospitalar faz-se pelo enfermeiro da triagem, aquando da existência de critérios de inclusão, e após avaliação da sintomatologia apresentada ${ }^{(16)}$. A tomada de decisão é facilitada pelos sinais clássicos da Escala de Cincinnati e pelos critérios constantes do protocolo específico (idade superior a 18 anos, início dos sintomas há menos de 4,5 horas e ausência de dependência prévia) ${ }^{(17,18)}$. Dos sinais da Escala de Cincinnati, um estudo de 2013 aponta a falta de força num dos membros como o sinal mais prevalente, seguindo-se a dificuldade em falar e a boca ao lado (27).

No presente estudo a informação inadequada relativamente ao tempo de manifestação dos sintomas foi a dificuldade mais referida pelos enfermeiros aquando a ativação do protocolo da VV do AVC. Sabese que a eficácia das terapêuticas de reperfusão para o AVC isquémico são tempo dependentes, e um dos objetivos da via verde passa precisamente pela redução dos tempos assistenciais ${ }^{(17,18)}$. No serviço de urgência, a eficácia da triagem e a rápida ativação do protocolo da VV influenciam o tempo porta-agulha. Contudo o tempo de início dos sintomas até à chegada ao serviço de urgência depende de múltiplas variáveis como sejam o rápido reconhecimento da situação e o pedido de ajuda diferenciada pela ativação dos serviços de emergência pré-hospitalar. Estas variáveis dependem em grande parte do doente, dos seus familiares e do público em geral, que devem estar capacitados a reconhecer a gravidade da situação e os sinais de alerta de $\operatorname{AVC}(17,18)$.

Este estudo apresenta algumas limitações que devem ser consideradas aquando da leitura e interpretação dos seus resultados. A principal delas consiste no facto de ter sido realizado com base numa amostra limitada de enfermeiros e apenas num serviço de urgência médicocirúrgica. Apesar desta limitação, e até onde chega o conhecimento bibliográfico dos autores, são raros em Portugal os estudos desenvolvidos sobre esta temática, pelo que o presente trabalho poderá constituir uma 
mais-valia e estimular o desenvolvimento de outros trabalhos de investigação sobre a avaliação e triagem do AVC.

\section{CONCLUSÃO}

A equipe de enfermagem tem papel central na triagem e avaliação inicial do doente com AVC na chegada ao serviço de urgência. O objetivo principal é fornecer assistência rápida e adequada contribuindo para a redução da mortalidade e incapacidade.

O presente estudo concluiu que, de um modo geral, os enfermeiros que realizam triagem não apresentam dificuldades significativas na avaliação e triagem de doentes com AVC. Uma análise mais pormenorizada revela contudo que em certas questões particulares poderá existir algum desconforto durante esta atividade assistencial, designadamente compaginar este processo com o receio em atrasar o acesso aos cuidados e a violência física e verbal por parte de utentes e familiares. Concretamente na ativação da VV do AVC o desconhecimento relativamente ao tempo de início dos sintomas foi a dificuldade mais referida pelos enfermeiros, pelo que se sugere mais educação em saúde de forma a consciencializar o público em geral para os sinais de alerta de AVC e apostar na melhoria dos processos de notificação e comunicação préhospitalar.

Recomendamos a realização de estudos multicêntricos em amostras mais representativas para entendermos melhor as dificuldades e constrangimentos na avaliação do AVC aquando da triagem, recordando que, particularmente no AVC isquémico, tempo é cérebro, e que as terapias de reperfusão atualmente disponíveis constituem elementos chave para a melhoria da sobrevivência, funcionalidade e qualidade de vida posterior ao evento cerebrovascular.

\section{REFERÊNCIAS BIBLIOGRÁFICAS}

1. Pannain GD, Ribeiro CC, Jacob MB, Pires LA, Almeida ALM. Relato de experiência: Dia Mundial do Acidente Vascular Cerebral. HU Rev. 2019;45(1):104-8.

2. Virani SS, Alonso A, Benjamin EJ, Bittencourt MS, Callaway CW, Carson AP, et al. Heart disease and stroke statistics-2020 update: A report from the American Heart Association. Circulation. 2020. 139-596 p.

3. Wang $Y$, Rudd AG, Wolfe CDA. Age and ethnic disparities in incidence of stroke over time: The South London stroke register. Stroke. 2013;44(12):3298-304.

4. Preto L, Dias Conceição M, Amaral SI, Figueiredo T, Ramos Sánchez A, Fernandes-Ribeiro AS. Fragilidad en ancianos que viven en la comunidad con y sin enfermedad cerebrovascular previa. Rev Cient la Soc Esp Enferm Neurol. 2017;46(c):11-7.

5. Oliveira A, Silva A, Sá N, Brandão S. Consulta de enfermagem de reabilitação ao doente pós evento cerebrovascular: Que desvios encontrados ao plano delineado à alta pelo enfermeiro de reabilitação? Rev Enferm Reabil. 2020;3(2):5-13.

6. INE. Instituto Nacional de Estatística - Causas de Morte: 2017 [Internet]. Causas de Morte 2017. 2019 [citado 25 de Outubro de 2020]. Disponível em: https://www.ine.pt/xurl/pub/358633033

7. DGS. Protocolo de Triagem de Prioridades em Urgência [Internet]. Despacho $n^{\circ} .19124 / 2005$ de 17 de Agosto. Circular Informativa $\mathrm{N}^{\circ}$ 52/DSPCS. 2005.2 Disponível em: http://www.grupoportuguestriagem.pt/

8. Camilo DGG, de Souza RP, Frazão TDC, da Costa Junior JF. Multi- criteria analysis in the health area: selection of the most appropriate triage system for the emergency care units in natal. BMC Med Inform Decis Mak. 2020;20(1):38.

9. Oliveira JL, Gatti AP, Barreto S, Junior JA, Góes HL, Matsuda LM. Acolhimento com classificação de risco: Percepç̃es de usuários de uma unidade de pronto atendimento. Texto Context Enferm. 2017;26(1):1-8.

10. Duro CLM, Lima MAD da S, Weber LAF. Nurses' Opinion on Risk Classification in Emergency Services. REME Rev Min Enferm. 2017;21:1-9.

11. Moreira DA, Tibães HBB, Batista RCR, Cardoso CML, Brito MJM. $O$ Sistema de Triagem de Manchester na atenção primária à saúde : Ambiguidades e desafios relacionados ao acesso. Texto Context Enferm [Internet]. 2017;26(2):1-8. Disponível em: http://dx.doi.org/10.1590/0104-07072017005970015

12. Carmo BA, Souza G. Atuação do enfermeiro na classificação de risco através do protocolo de manchester: uma revisão da literatura. Rev Eletrônica Acervo Saúde. 2018;11 (supl):1081-8.

13. Chabudé $T$, César $G$, Santana C. Acolhimento e Classificação de Risco em Unidade de Urgência: Relato de Experiência da Implantação do Sistema de Triagem de Manchester. Ensaios e Ciência. 2019;23(2):121-5.

14. Hermida PMV, Jung W, Nascimento ERP, Silveira NR, Alves DLF, Benfatto TB. Classificação de risco em unidade de pronto atendimento: Discursos dos enfermeiros. Rev Enferm. 2017;25(1):7-12.

15. Freitas MM. Dificuldades percecionadas e grau de Satisfação dos Enfermeiros que fazem Triagem de Manchester nos Serviços de Urgência. Instituto Politécnico de Leiria; 2014.

16. Barreira I, Martins MD, Silva NP, Preto P, Preto L. Resultados da implementação do protocolo da via verde do acidente vascular cerebral num hospital português. Rev Enferm Ref [Internet]. 2019;IV(22):117-26. https: / / doi.org/10.12707/RIV18085

17. Powers WJ, Rabinstein AA, Ackerson T, Adeoye OM, Bambakidis NC, Becker K, et al. Guidelines for the early management of patients with acute ischemic stroke: 2019 update to the 2018 guidelines for the early management of acute ischemic stroke a guideline for healthcare professionals from the American Heart Association/American Stroke A. Vol. 50, Stroke. 2019. 344-418 p.

18. Jauch EC, Saver JL, Adams HP, Bruno A, Connors JJB, Demaerschalk BM, et al. Guidelines for the early management of patients with acute ischemic stroke: A guideline for healthcare professionals from the American Heart Association/American Stroke Association. Stroke. 2013;44(3):870-947.

19. Pereira MSM, Guedes HM, Oliveira LM, Martins JC. Relação entre o Sistema de Triagem de Manchester em doentes com AVC e o desfecho final. Rev Enferm Ref [Internet]. 2017;13(IV):93-102. Disponível em: https://doi.org/10.12707/RIV16079

20. Hill MM, Hill A. Investigação por Questionário. $2^{\mathrm{a}}$ Edição. Sílabo E, editor. 2012.

21. Salgueiro ACV. Triagem de Manchester: satisfação dos enfermeiros [Internet]. Instituto Politécnico de Viseu; 2019. Disponivel em: http://hdl.handle.net/10400.19/5649

22. Donoso MTV. 0 género e suas possíveis repercussões na gerência de enfermagem. Rev Min Enf. 2000;4(1/2):67-9.

23. Amaral PMF. Qualidade da Triagem de Manchester e fatores que a influenciam. Escola Superior de Enfermagem de Coimbra; 2017.

24. Souza C, Araújo FA, Chianca TCM. Scientific literature on the reliability and validity of the manchester triage system (MTS) protocol: A integrative literature review. Rev da Esc Enferm da USP. 2015;49(1):144-51.

25. Correia JAC. Violência no trabalho dos enfermeiros no serviço de urgência. Instituto Politécnico de Viana do Castelo; 2016.

26. Martins HG. Violência contra os profissionais de saúde nos serviços de urgência. Instituto Politécnico de Viseu; 2018.

27. Moutinho M, Magalhães R, Correia M, Silva C. Avaliação da Via Verde do Acidente Vascular Cerebral no Norte de Portugal: Caracterização e Prognóstico dos Utilizadores. Acta Med Port. 2013;26(2):113-22. 